\title{
THE SURFACE ACTIVITY OF POLYMERS IN COSOLVATED SYSTEMS DETERMINED FROM COMPUTATIONAL SIMULATION
}

\author{
Guy Francis Mongelli \\ Department of Chemical Engineering, Case Western Reserve University \\ 10900 Euclid Ave., A.W. Smith 116, Cleveland, OH 44120 \\ Gfm12@case.edu
}

\begin{abstract}
The present body of literature fails to adequately address the need for simulation metrics which determine the surface activity of target species in single or multi-solvent systems. Within this work a new property determinable by computational molecular dynamics simulation is detailed. That is the surface parameter, which is an indicator of the placement of a molecule with respect to the interface. Simulations were carried out on polyalkanes in alcohol co-solvated systems to verify systematically that the OPLS-AA force fields can accurately describe the solvation of low molecular weight polyalkanes with increased alcohol content.This parameter and the associated codeare helpful in identifying quickly if a molecule is surface active, especially in mixed solvent systems. The code for the determination of this property is written in FORTRAN, a widespread and readily available package on most supercomputing systems where GROMACS is deployed.
\end{abstract}

\section{Introduction}

Simulations of liquid molecular behavior and associated properties have become possible through the use of modern and high performance computing packages ${ }^{1}$. While such packages offer the capability to determine many quantities of interest such as radius of gyration, radial distribution function, density, hydrogen bonding ${ }^{2}$, and relative ordering, such simulations did not indicate the solubility properties nor their surface active capability ${ }^{3}$, dubbed surfactantability, of such simulated materials.

This manuscript addresses such a lacking in describing a rigorous, mathematical computation to determine at each individual simulation time step and cumulatively, the position of a molecule or molecules of interest relative to bulk solvent molecules. Molecules with these surface active properties such as polyethylene, polypropylene, polyisobutylene, polymethylene oxide, polyethyleneoxide, polypropyleneoxide, and polydimethylsiloxane have broad commercial interest in the shampoo and shaving cream industries according to theSpecialty Surfactants Market - Global Scenario, Raw Material And Consumption Trends, Industry Analysis, Size, Share \& Forecast 2011 - 2017 (http://www.prnewswire.com/news-releases/specialty-surfactantsindustry-is-expected-to-reach-usd-292-billion-globally-in-2017-transparency-market-research161943735.html), and Dow Corning's articles, "What makes silicones special?" (http://www.dowcorning.com/content/discover/discoverchem/si-vs-organic.aspx) and "Fascinating silicone for beauty and personal care" (http://www.dowcorning.com/content/discover/discovershowcase/personal.aspx). Mixed solvent systems have been recently simulated and are of particular interest ${ }^{4}$.

New methods are continually under development to probe the structure of molecules at the interfaces ${ }^{5}$. It is often helpful to have computational methods to determine quantities 


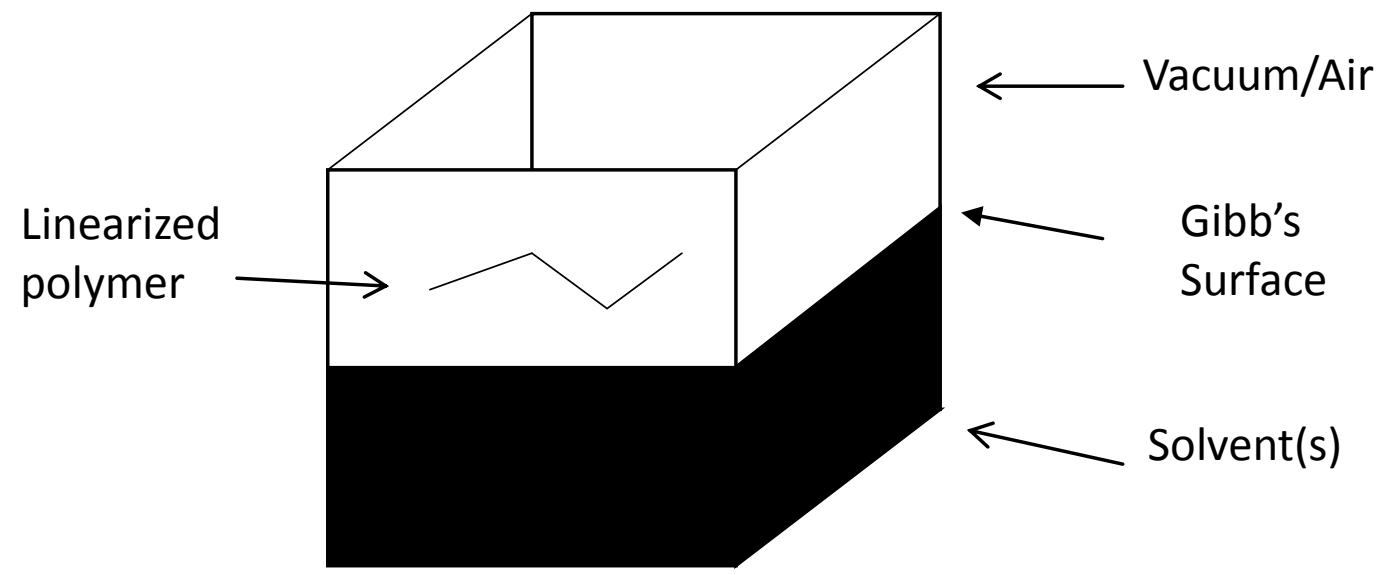

Figure 1: A depiction of the initial state of the system when performing NVT ensemble simulations of polymers in co-solvated systems. Within this figure, the four carbon polymer would have a first surface parameter of 1 , since all of its atoms are above the solvent atoms.

typically performed through experiment. Computational molecular dynamics may in such a manner strive to predict structure property relationships before an entire class of molecules is synthesized, the approach Vaney and Voight have taken ${ }^{6}$.

\section{Methods}

In order to ascertain the surface activity of polymers in co-solvated systems, a FORTRAN program was written to determine the coordinates of polymer atoms relative to those of solvent atoms. The fraction of the simulation time that polymer solute atoms spend above the solvent atoms is the time-averaged surface parameter, which is derived from the instantaneous surface parameter and in turn, the number of solvent atoms above and below each of the polymer atoms with logic functions.

Generally, the surface parameter seeks to link together concepts regarding occupied space for the solutes relative to the solvents and the relative energies of intermolecular interactions between relevant species of interest.

The FORTRAN program ${ }^{7}$ determines five different surface parameter values, only the first of which is plotted in this manuscript. The fifth $(n B o t)$ is the instantaneous single frame fraction of solvent molecules beneath the polymer, the fourth (nTop) is instantaneous single frame fraction of solvent molecules above the polymer, the third $(\overline{n B o t})$ is the nth step average of the $n B$ ot value from the first frame, the second (f2) is nth step average of the $n T o p$ value from the first frame, the first (f) is nth step average of the fraction of the polymer atoms above the solvent from the first step. As a self-consistency check, a nested logic function, $=I F(E 3>0, I F(F 3>0,1,0), 0)$, is evaluated to determine if the $f 4$ and $f 5$ values are simultaneously non-zero a potential indicator that there is a simulation error. If true, then a value of 1 is returned, if false a value of zero is returned.Such a logic number is computed for each of the atoms within the polymer molecule, and these are averaged to create the second surface parameter value, or the instantaneous surface parameter. Such instantaneous values are then time-averaged to generate the first surface parameter value. This first surface parameter value is what is depicted in the plots. These simulations have FORTAN surface analysis outputs which are self-consistent as indicated by false outputs for each of the nested logic functions for all 
t.The poly-alkanes are numbered according to the total number of carbons present in the molecule. An alternative naming scheme involves naming by the number of backbone sites, the number of hydrogens, and the molecular weight; all of which map one-to-one onto the 3D structures.

Discussion

It is noteworthy that once the polymer is solvated, it tends to not emerge from the bulk and enter a large valued surface parameter or gaseous state. In these simulations the extent of equilibration is determined by the convergence of the surface parameter to a steady value that does not change for several nanoseconds. In cases where the surface parameter decreases and then increases again, especially in low alcohol contents, there is likely a spontaneously and locally increase alcohol concentration in the vicinity of the interface, which attracts the polymer down. Since such a state is not an equilibrium state, the increased content deforms, and the polymer returns to the surface ${ }^{2}$.

On defining equilibration

The average and standard deviations of changes in the ensemble potential, density profile, surface parameter and other downstream computations of interest should be utilized in conjunction to determine when equilibrium simulations should terminate. In other words, when the single or combined profiles of the downstream computations of interest reach a certain minimum change threshold, the simulation is set to terminate and potentially email the user. Within the context of these ensembles, the longest quantity to equilibrate is the surface parameter, which takes approximately 25 nanoseconds. Again, these simulations are 25 nanoseconds. In the context of the simulations presented within this thesis the equilibration criterion is simpler than ideal, in the sense that equilibration is assumed when the timeaveraged surface parameter and density no longer change with increased simulation properties.At equilibrium, the slab thicknesses for $00 \%, 50 \%, 70 \%$ and $100 \% \mathrm{EtOH}$ are respectively $4.5,5.7,8.7$, and $11.7 \mathrm{~nm}$ respectively.

Other concepts of equilibration include uniqueness of ensemble properties, steady state, thermal equilibrium with other systems, maximized entropy, and minimized free energy. The free energy of such systems and entropies have not been determined for these systems of interest and therefore their extent of equilibration has not been quantified.

Some alternative checks for ensemble correctness include testing the first law of thermodynamics, observing pressure change and energy fluctuations scaling with $\mathrm{N}$, the number of atoms. One goal of molecular dynamics is to have the changes in all of the observable properties of the system have fluctuations of less than $5 \%$, which is not true in the cases of pressure tensors for systems of this scale. Hydrogen bonding, density, surface parameter all indicate that these simulations are equilibrated however. Hydrogen bonding reaches equilibration on a time scale which is an order of magnitude shorter than the surface parameter does for these systems, i.e. 1 ns vs. ca. 25 ns.

Results

In each of these polymers and alcohol contents, the surface parameter indicates that the polymer of prefers to be at the interface most of the simulation time. PIB 24C is an exception in the $70 \% \mathrm{EtOH}$ content. It is not clear yet the molecular weight dependence of the solubility of these polymers and how the free energy of solvation depends on the chain length. 
Once such parameters are calculated, the pattern and causes for the trends may become clearer.

This indicates that the interactions with the solvent mixture are unfavorable and that these polymers will impact the surface tension of the mixture. Since the instantaneous surface parameter values are weighted smaller as the simulation progresses and equilibration of the system can lead to a thermodynamic state significantly different from the initial state of the system, the surface parameters were averaged for thousand step intervals.

It is possible to "turn off" certain types of molecular interactions and in doing so determine which types of interactions contribute to observed molecular behavior. In several studies, the dihedral interactions were turned off to determine their impact on the surface activity of poly-alkane compounds. Note that the $U$ and Coulombic interactions may still impose significant restrictions upon the torsional strains of the system. These cannot be turned off without causing the simulation to "blow up", since the $\sqcup$ forces cause repulsions between Coulombically attractive species nuclei. Although, the restrictions imposed by Coulombics on the polymeric torsional space in mixed solvents can be readily studied.

One method to determine which aspects of molecular characteristics -- an essential approach in molecular design -- is to determine what contribution particular force field potentials make towards affecting critical properties derived via downstream computations. This can be accomplished through alteration of the molecular topologies and in some cases complete removal of force field potentials from the ensemble.

The dihedral energy for each atom bonded in a molecule can be determined from the force field parameter class mappings of all immediately bonded four atom groupings. The theoretical fraction of the angular space associated with dihedral interaction energies below $\mathrm{k}_{\mathrm{B}}{ }^{*} \mathrm{~T}$ at each atom can be calculated with simple algebraic and calculus techniques. In such a manner, the theoretical fraction of an entire molecules' configurational space it is exploring at a given temperature can be determined. These entropy trends compared to the entropy differences observed from GROMACS potentials.

For the polymers studies in this manuscript, the energies of rotation are below kB*T. In other words, such differences are not resulting in glassy polymers or exhibiting liquid crystalline behavior in these mixtures. In a separate calculation, the entropy of polymers at bulk states and interface states in slab systems can be determined by correlating manipulated ensemble potentials to the surface parameter.

The distances between oxygens, carbons, hydrogens, or silicons due to bond lengths, hybridizations and dihedral interactions are not specific enough nor restrictive enough relative to the Coulombic interaction energies of the system. $U$ forces are attractive then repulsive between all atoms depending on how close the atoms are to each other. However, Coulombic interactions are either attractive or repulsive independent of position. In order to come up with an understanding of what atomic parameters result in differences in solvophilicities, we will need to look at which forces can result in forces that will be specific to differences in the atoms in the variables that change as a function of mass content of co-solvents. Since, $\sqcup$ forces do not see large variations in space and between different types of atoms, it is much more likely that the Coulombic interactions will dictate solvophilicity. $\mathrm{H}$ bonding is simply a metric if the $\mathrm{LJ}$ and Coulombic forces are strong enough to bring certain types of atoms close together for long 
periods of time. Such bonding indicates the relative destabilization of the system from placement of the polymer in the bulk.

To support these claims, we will observe the surface parameter of PE12 vs. PP12 vs. PIB12. All of these have the same numbers of carbon atoms, yet different placements. Admittedly, they have different numbers of hydrogens, however the charges on these atoms are smaller than in carbons or oxygen systems, so they will result in smaller Coulombic charge by at least a factor of 2 in $\mathrm{CH} 2$ or 3 for $\mathrm{CH} 3$. Therefore, at the same distance, the forces resulting will be a factor of 4 or 9 smaller with respect to an arbitrary or fundamental charge. Table I: The following table indicates the average surface parameter (f) over the entire simulation for poly-alkane polymers of initial interest in pure aqueous systems. The surface parameter is the simulation-time-average fraction of the polymer atoms which have no solvent molecules above them. Dihedral interactions were removed from the ensemble computations and did not affect these values. This indicates that the lack of solubility of such polymers is caused by unfavorable interactions with the solvent and not dihedral-entropic restraints. Note that when re-running simulations, the surface parameters is subject to some small variations.

\begin{tabular}{|c|c|c|}
\hline & $\begin{array}{c}\text { Dihedrals Off } \\
\text { Surface Parameter, } \mathbf{f}\end{array}$ & $\begin{array}{c}\text { Dihedrals On } \\
\text { Surface Parameter, } \mathbf{f}\end{array}$ \\
\hline PE - 12C & 0.935 & 0.956 \\
\hline PE - 18C & 0.983 & 0.984 \\
\hline PIB - 24C & 0.988 & 0.988 \\
\hline PP - 15C & 0.974 & 0.971 \\
\hline PP - 18C & 0.977 & 0.969 \\
\hline PP - 24C & 0.935 & 0.97 \\
\hline
\end{tabular}

Figure 2: The following graphs indicate the average surface parameter (f1) over the entire simulation for polyalkane polymers of initial interest in a mixture of $50 \%$ by mass solution of $\mathrm{EtOH}$ and water:
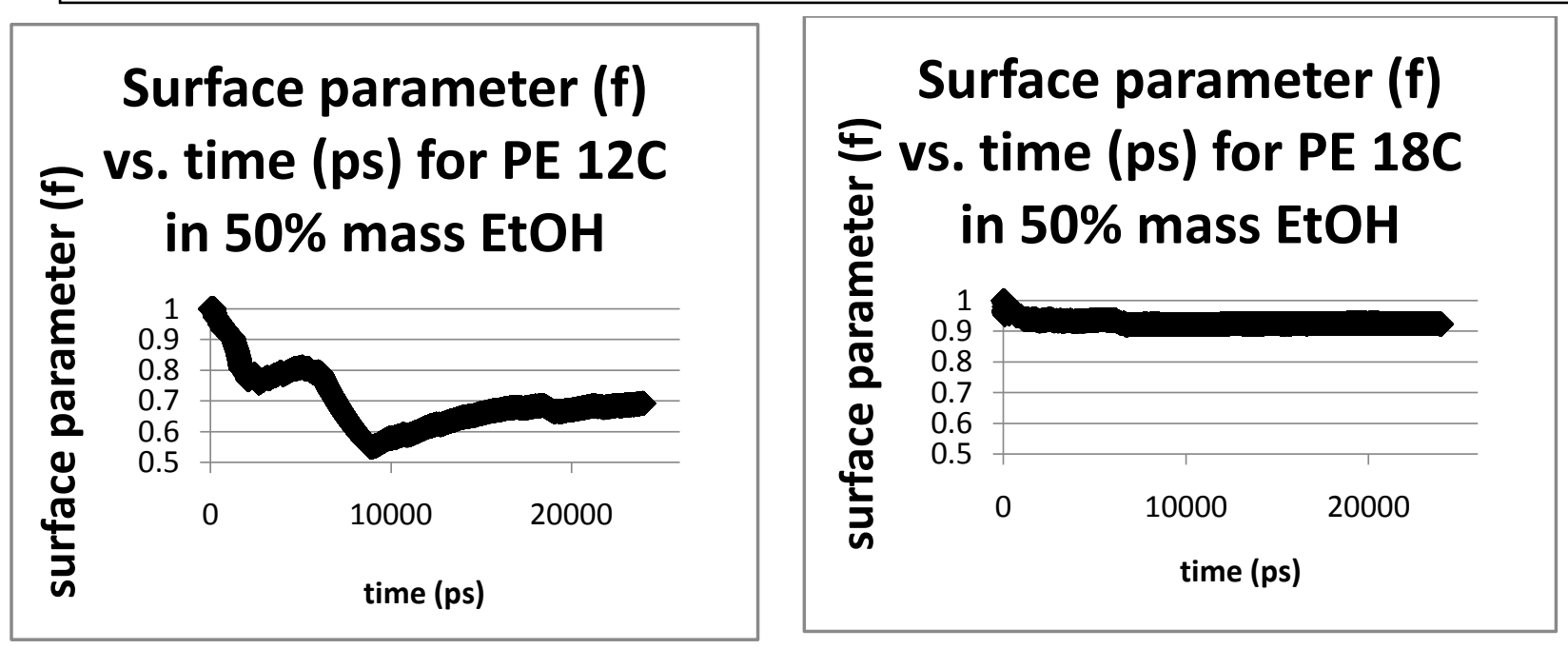

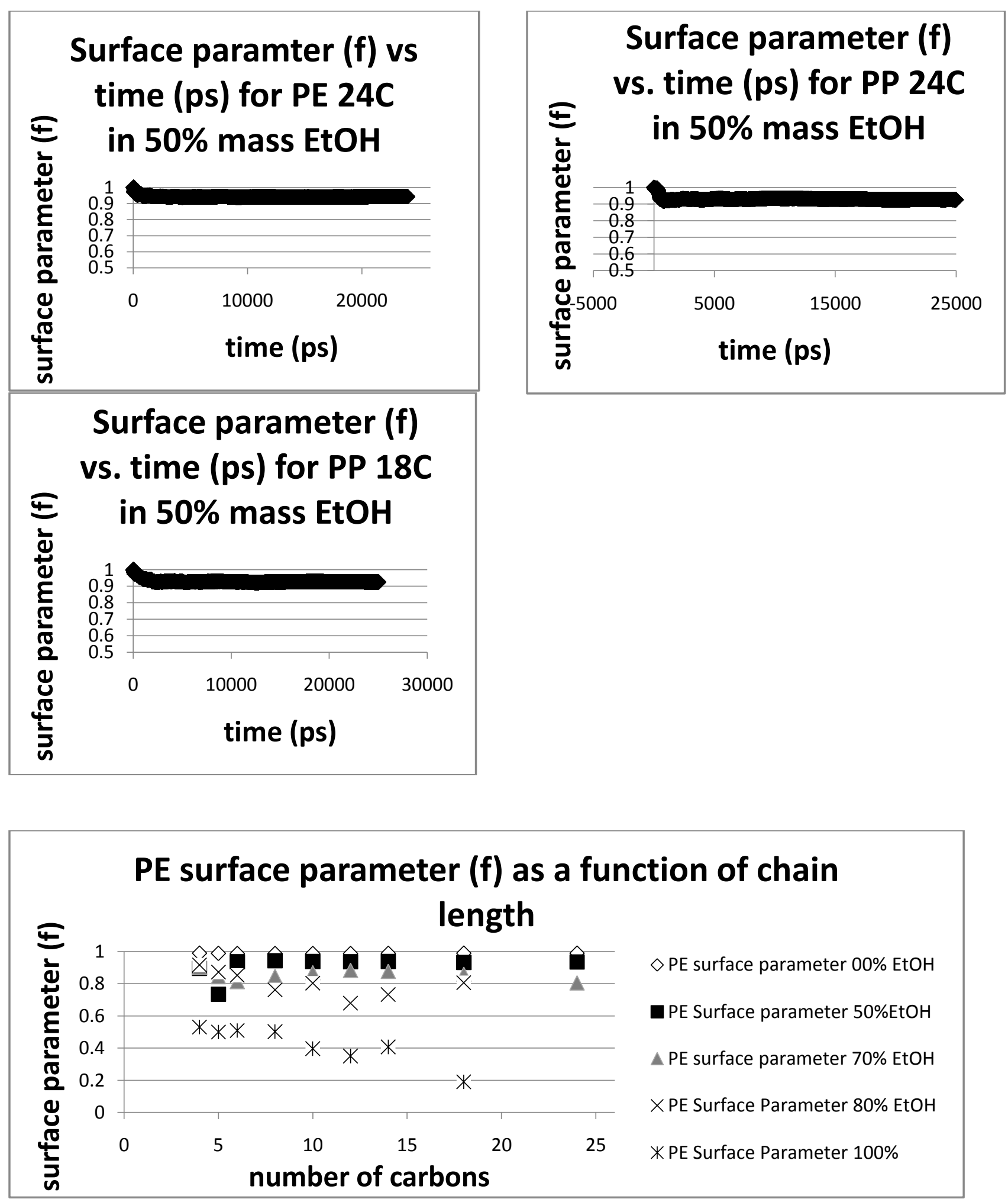

Figure 3: The surface parameters of polyethylenes as a function of alcohol content for multiple chain lengths. 


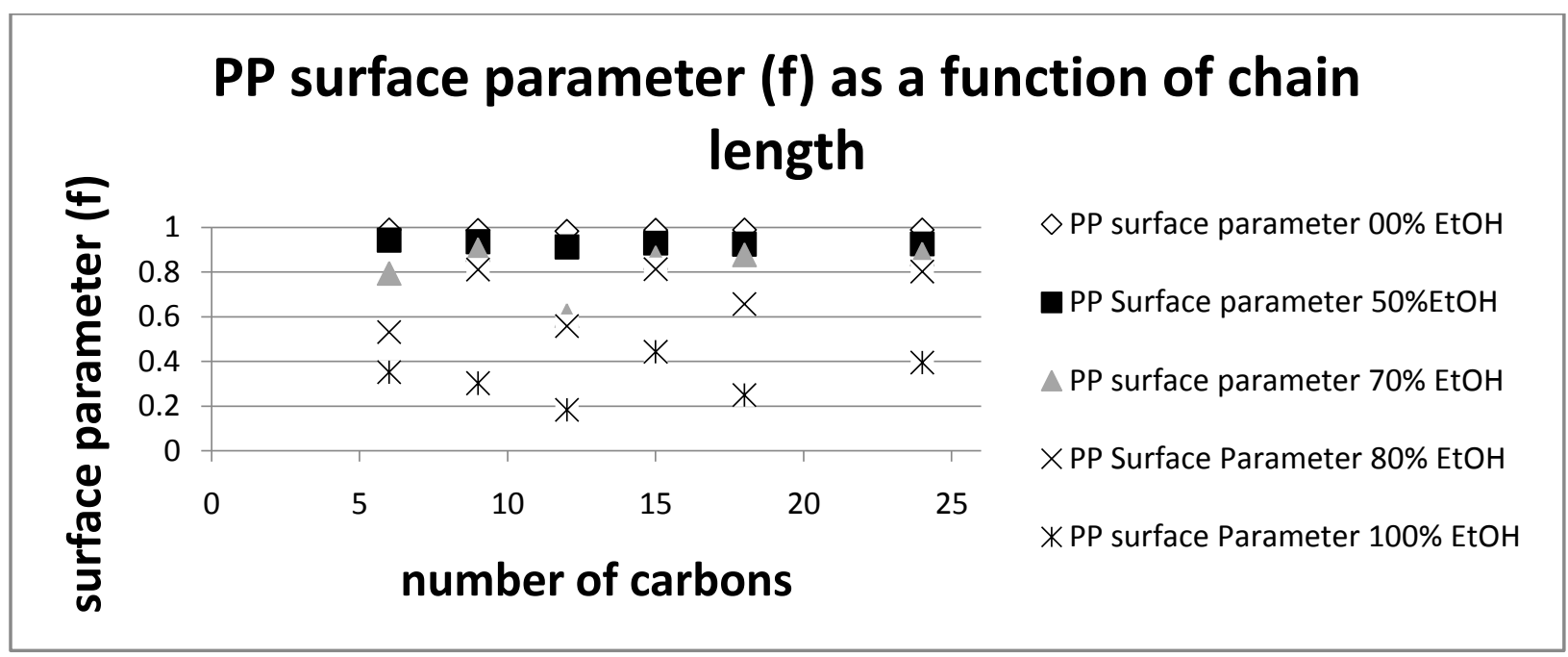

Figure 4: The surface parameter of polypropylenes as a function of alcohol content for multiple chain lengths.

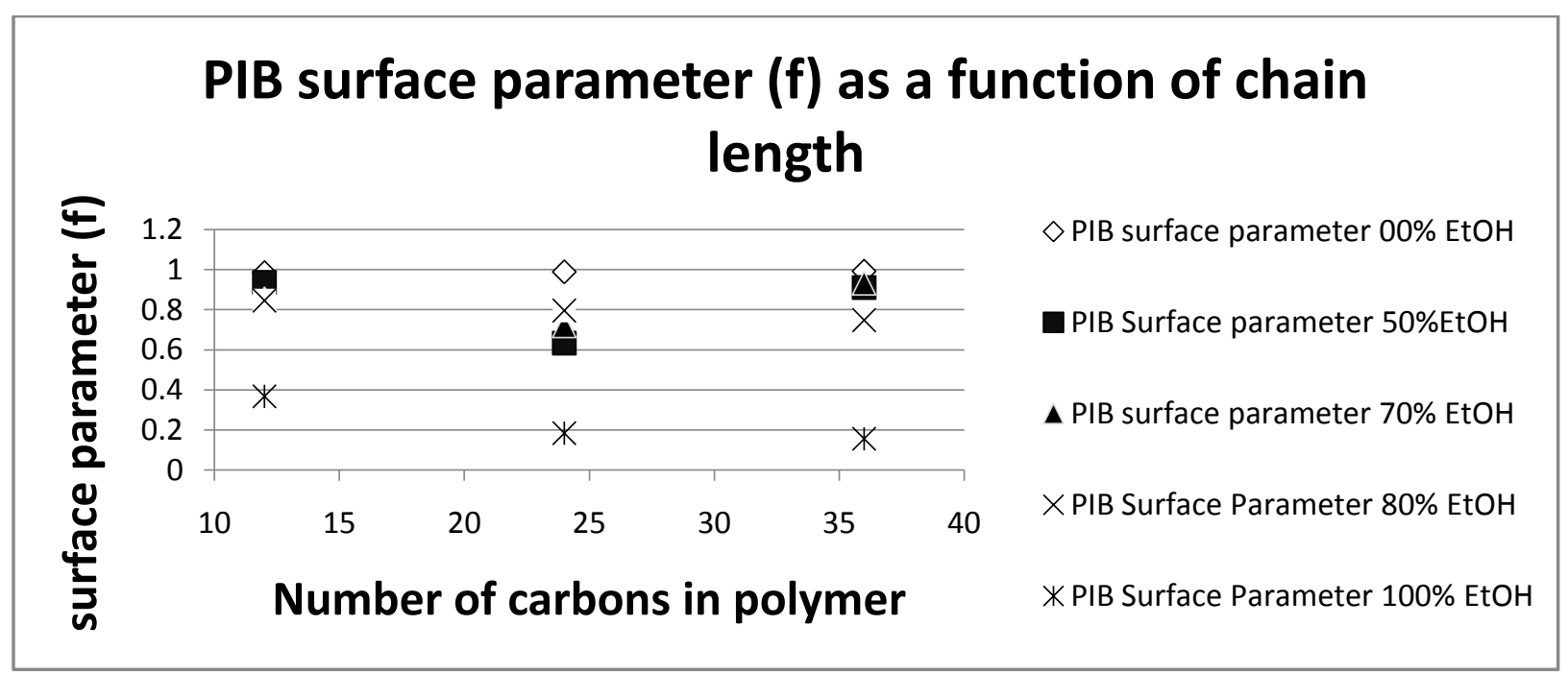

Figure 5: The surface parameter of polypropylenes as a function of alcohol content for multiple chain lengths.

\section{Conclusions}

A new quantity of great interest in classifying the surface activity of polymers in co-solvated mixtures was introduced. The newly presented computational method to determine surfactantability makes it possible to determine the usefulness of yet synthesized molecules in established solvents. Within this manuscript, the surface parameter of polyethylenes, polypropylenes, and polyisobutylenes of low molecular weight were simulated in various ethanol contents. The surface parameter decreases overall in all cases with increased alcohol content. In the case of polyethylenes and polypropylenes, the surface parameter increased as a function of chain length. In polyisobutylenes, the surface parameter generally decreased as a function of chain length. In several alcohol contents, there was an increase, and this is likely due to the nature of how alcohol is added to co-solvated systems and weather it adds to the 
surface or the bulk, and is within the $1 \mathrm{~nm}$ distance required for intermolecular interactions via non-bonded forces to be significant.

\section{Acknowledgements}

I would like to acknowledge the National Science Foundation Award Abstract \#1159327.

\section{References}

${ }^{1}$ S. Pronk, et al.; "GROMACS 4.5: a high-throughput and highly parallel open source molecular simulation toolkit"Bioinformatics 29 845-854(2013).

${ }^{2}$ D. Bedrov and G. D. Smith, "A molecular dynamics simulation study of the influence of hydrogenbonding and polar interactions and polar interactions on hydration and conformations of a poly(ethylene oxide) oligomer in dilute aqueous solution", Macromolecules 35, 5712 (2002).

${ }^{3} \mathrm{M}$. Kinoshita, "Interaction between surfaces with solvophobicity or solvophilicity immersed in a solvent" J. Chem. Phys. 118, 8969 (2003).

${ }^{7}$ L. Dougan, S.P. Bates, R. Hargreaves, J.P. Fox, J. Crian, J.L. Finney, V. Réat, A.K. Soper; "Methanol Water Solutions: A bi-percolatingliquid mixture" J. Chem Phys. 1221, 6456 (2004).

${ }^{8}$ E.K. Mann, L.T. Lee, S. Henon, D. Langevin, J. Meunier "Polymer Surfactant Films at the Air-Water Interface" Macromolecules 26, 25 7037-7045 (1993).

${ }^{9}$ R.H. Baney, C.E. Voight, J.W. Mantelle "Structure-Solubility Relationships in Polymers" Ed. H. Seymour Academic Press, NY (1977).

${ }^{10}$ http://hpcadventures.blogspot.com/2014/05/a-fortran-program-towards-surface.html on hydration and conformations of a poly(ethylene oxide) oligomer in dilute aqueous solution", Macromolecules 35, 5712 (2002).

${ }^{11} \mathrm{G}$. Jimenez-Serratos, C. Vega, A. Gil-Villegas; "Evaluation of the pressure tensor and surface tension for molecular fluids with discontinuous potentials using the volume perturbation method" J. Chem. Phys. 137, 204104 (2012). 